\title{
Psychological status is associated with drug efficacy in patients with ankylosing spondylitis
}

Background: Quality of life in patients with Ankylosing Spondylitis (AS) is significantly associated with psychological disorders, such as depression and anxiety. Effective prevention and control of depression and anxiety in AS patients depend on important advances in our comprehensive understanding of its risk factors. However, whether psychological disorders in AS patients are related to drug efficacy is unclear. For this reason, the objective of this study was to explore the association between psychological status and drug efficacy in AS patients. Methods: A total of 3,147 AS patients were recruited from outpatient departments of 12 general hospitals in China. The level of anxiety disorder was evaluated with the Zung Self-Rating Anxiety Scale and the Zung Self-Rating Depression Scale was used to evaluate the level of depression. The association between psychological status and drug efficacy was analyzed for overall and different drugs. Results: The mean anxiety index was significantly $(p<0.001)$ lower in AS patients in efficacy group $(0.474 \pm 0.004)$ than patients in inefficacy group $(0.512 \pm 0.005)$. The mean depression index was significantly $(p<0.001)$ lower in AS patients in efficacy.

Keywords: ankylosing spondylitis • psychological status $\cdot$ NSAIDs $•$ sulfasalazine

\section{Abbreviations}

AS: Ankylosing Spondylitis; SAS: Zung Selfrating Anxiety Scale; SDS: Zung Self-rating Depression Scale; NSAIDs: Non-Steroid AntiInflammatory Drugs; SSZ: Sulfasalazine; TNF:

Tumor Necrosis Factor

\section{Introduction}

Ankylosing Spondylitis (AS) is a chronic inflammatory disease characterized by inflammatory back pain that can lead to structural and functional impairments [1]. The mean prevalence of AS is 7.4 to 31.9 per 10,000 [2]. AS increases the risk of mortality [3]. AS imposes substantially direct and indirect costs to individuals, families, and societies $[4,5]$. In addition to economic costs, AS has a long-term negative impact on the quality of life [6].

Due to the progress in early diagnosis and efficient therapy, the prognosis of AS has been dramatically improved over the past decades, therefore, greater focus now is on the patient's quality of life [7,8]. Quality of life in patients with AS is significantly associated with psychological disorders, such as depression and anxiety [9-11]. Disease activity and functional disability are the main risk factors of psychological disorders in patients with AS [12]. In addition, low socioeconomic status, lack of health insurance and fatigue contribute to psychological disorders in patients with AS $[11,13]$.

Effective prevention and control of depression and anxiety in patients with AS depend on important advances in our comprehensive understanding of its risk factors. There is high prevalence of psychological disorders in patients with other drug-refractory chronic disease [14]. These patients' psychological disorders can be significantly improved after effective therapy $[15,16]$. Although Non-Steroid AntiInflammatory Drugs (NSAIDs) and Tumor Necrosis Factor (TNF) $\alpha$ blockers are effective, not all patients achieve remission or a major clinical response [17]. However, whether psychological disorders in patients with AS are related to drug efficacy is unclear. For this reason, the objective of this study was to explore the association between psychological status and drug efficacy in patients with ankylosing spondylitis.

\section{Patients and methods}

\section{Patients}

Patients with AS were recruited from out-patient

\author{
Yanli Zhang, Zetao Liao, \\ Yutong Jiang, Zhiming Lin, \\ Mingcan Yang, Qing Lv, Qiujing \\ Wei, Shuangyan Cao \& Jieruo \\ $\mathbf{G u}^{*}$ \\ Department of Rheumatology, Third \\ Affiliated Hospital of Sun Yat-Sen \\ University, Guangzhou, China \\ *Author for correspondence: \\ gujieruo@163.com
}


departments of 12 general hospitals in China between November 2015 to October 2017. Patients were included if they met the Modified New York criteria for AS [18]. Patients were excluded if

- They coexisted with other rheumatic diseases, malignancies, central nervous system diseases, chronic kidney diseases, chronic liver diseases and thyroid diseases.

- They were pregnant. This study was approved by the Ethics Committee of the Third Affiliated Hospital of Sun Yat-sen University. All patients signed informed written consent forms.

\section{Data collection}

Demographic, psychological status and prescription medication information were collected by trained interviewers according to a pre-established questionnaire from November 2015 to October 2017.

\section{Psychological status assessment}

The level of anxiety disorder was evaluated with the Zung Self-Rating Anxiety Scale (SAS). SAS is a 20-item self-administrated scale to measure anxiety based on scoring in 4 groups of manifestations: cognitive, autonomic, motor and central nervous system symptoms [19]. The Zung Self-Rating Depression Scale (SDS) was used to evaluate the level of depression. SDS is also a 20 items on the scale that rate the rating affective, psychological and somatic symptoms associated with depression [20]. The scores greater than 45 indicate anxiety or depression.

Pharmacologic therapy and efficacy of therapy

The prescription medication information and efficacy of therapy were collected in interview by self-reported. Prescription medications included Non-Steroidal Anti-Inflammatory Drugs (NSAIDs), sulfasalazine (SSZ) and anti-Tumor Necrosis Factor (TNF) agent Eenbrel. NSAIDs continued were defined as using NSAIDs for more than 12 weeks. The efficacy of therapy was defined as Pain (visual analog scale (VAS) and Patient Global Assessment of Disease Activity (PGA) improved by at least $20 \mathrm{~mm}$. The efficacy of NSAIDs was assessed 2 weeks after using them. The efficacy of SSZ and Enbrel were assessed 12 weeks after using them.

\section{Statistical analysis}

All statistical analyses in this study were performed using SPSS software (version 18.0). Continuous variables were presented as means with standard errors; categorical variables were presented as percent. Tests of significance were performed using t-test for continuous variables and chi-squared tests for categorical variables. In addition, linear regression models were performed to evaluate the association between anxiety or depression and the efficacy of therapy after adjusting for sex, age, age of onset and different drugs. A two-sided p-value less than 0.05 were considered to be statistically significant. Pearson correlation analysis was performed to evaluate the association between anxiety and regression.

\section{Results}

Demographic characteristics and drug therapy status between drug efficacy group and drug inefficacy group among patients with AS

A total of 3,147 patients with AS were included in the study. Of the 3,147 patients, 1,441 (45.8\%) patients were reported to be efficacy and 1,706 (54.2\%) participants reported to be inefficacy. Demographic characteristics and drug therapy status were listed in Table 1 . There were no significant differences in gender, age group and age of onset between these two groups. There was a significant difference in drug therapy status between these two groups. For example, the efficacy group had higher proportions of drug therapy status than the inefficacy group for NSAIDs on demand (32.8\% vs $22.2 \%)$, NSAIDs continued $(49.8 \%$ vs $33.2 \%)$, SSZ $(54.9 \%$ vs $27.6 \%)$ and biologic agents $(12.8 \%$ vs $8.6 \%)$.

Anxiety and depression index between drug efficacy group and drug inefficacy group among patients with AS

The mean anxiety index was significantly $(\mathrm{p}<0.001)$ lower in AS patients in efficacy group (mean \pm standard error: $0.474 \pm 0.004$ ) than AS patients in inefficacy group (mean \pm standard error: $0.512 \pm 0.005)$. The difference was significant for NSAIDs on demand, NSAIDs continued and SSZ between these two groups (Table 2).

The mean depression index was significantly $(\mathrm{p}<0.001)$ lower in AS patients in efficacy group (mean \pm standard error: $0.532 \pm 0.003$ ) than AS patients in inefficacy group (mean \pm standard error: $0.566 \pm 0.004)$. The difference was significant for NSAIDs on demand, NSAIDs continued and SSZ between these two groups (Table 2). 
Psychological status is associated with drug efficacy in patients with ankylosing Research Article spondylitis

\begin{tabular}{|c|c|c|c|c|c|}
\hline & \multicolumn{2}{|c|}{ Efficacy group ( $N=1441$ ) } & \multicolumn{2}{|c|}{ Inefficacy group ( $N=1706)$} & \multirow[b]{2}{*}{ p value } \\
\hline & $\mathbf{N}$ & Percent or mean $( \pm$ SE) & $\mathbf{N}$ & Percent or mean $( \pm$ SE) & \\
\hline \multicolumn{6}{|l|}{ Gender } \\
\hline Male & 1149 & $79.7 \%$ & 1366 & $80.0 \%$ & \multirow{2}{*}{0.8157} \\
\hline Female & 292 & $20.3 \%$ & 340 & $20.0 \%$ & \\
\hline \multicolumn{6}{|l|}{ Age group (years) } \\
\hline $0-19$ & 311 & $21.6 \%$ & 324 & $19.0 \%$ & \multirow{4}{*}{0.0910} \\
\hline $20-39$ & 988 & $68.6 \%$ & 1174 & $68.8 \%$ & \\
\hline $40-59$ & 135 & $9.3 \%$ & 197 & $11.6 \%$ & \\
\hline$\geq 60$ & 7 & $0.5 \%$ & 11 & $0.6 \%$ & \\
\hline \multicolumn{6}{|l|}{ Therapy } \\
\hline NSAIDs on demand & 473 & $32.8 \%$ & 378 & $22.2 \%$ & $<0.0001$ \\
\hline NSAIDs continued & 718 & $49.8 \%$ & 567 & $33.2 \%$ & $<0.0001$ \\
\hline SSZ & 791 & $54.9 \%$ & 471 & $27.6 \%$ & $<0.0001$ \\
\hline Etanercept & 185 & $12.8 \%$ & 146 & $8.6 \%$ & 0.0005 \\
\hline Age of onset & 1441 & $20.9 \pm 0.19$ & 1706 & $21.4 \pm 0.18$ & 0.1379 \\
\hline
\end{tabular}

Table 2. Anxiety and depression index between efficacy group and inefficacy group among patients with ankylosing spondylitis.

\begin{tabular}{|c|c|c|c|c|c|}
\hline & \multicolumn{2}{|c|}{ Efficacy group (N=1441) } & \multicolumn{2}{|c|}{ Inefficacy group (N=1706 ) } & \\
\hline & $\mathbf{N}$ & Mean $\mathbf{( \pm \mathbf { S E } )}$ & $\mathbf{N}$ & Mean $\mathbf{( \pm \mathbf { S E } )}$ & p value \\
\hline Anxiety index & & & & & \\
\hline Overall & 1441 & $0.474 \pm 0.004$ & 1706 & $0.512 \pm 0.005$ & $<0.0001$ \\
\hline NSAIDs on demand & 473 & $0.481 \pm 0.008$ & 378 & $0.520 \pm 0.010$ & $<0.0001$ \\
\hline NSAIDs continued & 718 & $0.462 \pm 0.005$ & 567 & $0.495 \pm 0.008$ & $<0.0001$ \\
\hline SSZ & 791 & $0.474 \pm 0.006$ & 471 & $0.502 \pm 0.009$ & $<0.0001$ \\
\hline Etanercept & 185 & $0.459 \pm 0.010$ & 146 & $0.460 \pm 0.012$ & 0.8574 \\
\hline Depression index & & & & & \\
\hline Overall & 1441 & $0.532 \pm 0.003$ & 1706 & $0.566 \pm 0.004$ & $<0.0001$ \\
\hline NSAIDs on demand & 473 & $0.533 \pm 0.007$ & 378 & $0.562 \pm 0.009$ & $<0.0001$ \\
\hline NSAIDs continued & 718 & $0.524 \pm 0.005$ & 567 & $0.552 \pm 0.007$ & 0.0026 \\
\hline SSZ & 791 & $0.532 \pm 0.005$ & 471 & $0.559 \pm 0.008$ & 0.0126 \\
\hline Etanercept & 185 & $0.516 \pm 0.009$ & 146 & $0.526 \pm 0.011$ & 0.5673 \\
\hline
\end{tabular}

Anxiety and depression index were associated with efficacy of drug therapy among patients with AS

We employed linear regress models to estimate the associations between anxiety or depression and the efficacy of drug therapy adjusting for sex, age group, age of onset, and drug therapy status (NSAIDs on demand, NSAIDs continued, SSZ and biologic agents). Compared with the inefficacy group, the anxiety index would decrease 0.0315 (95\% CI: 0.0180-0.0450, $\mathrm{p}<0.0001)$ in efficacy group for patients with AS after adjusting for co-variables. Compared with the inefficacy group, the depression index would decrease 0.0269 (95\% CI: $0.0148-0.0390$, $\mathrm{p}<0.0001$ ) in efficacy group for patients with AS after adjusting for co-variables (Table 3).

Anxiety index was associated with depression index

Using Pearson correlation analysis, wefound strong association between anxiety index and depression index (correlation coefficient: 0.851, Figure 1).

\section{Discussion}

To our knowledge, this is the first study to examine the association between psychological status and drug efficacy in patients with ankylosing spondylitis. AS patients in drug 


\begin{tabular}{|c|c|c|c|c|}
\hline Parameter & Estimate & \multicolumn{2}{|c|}{$95 \% \mathrm{Cl}$} & $p$ value \\
\hline \multicolumn{5}{|c|}{ For anxiety } \\
\hline Efficacy & -0.0315 & -0.045 & -0.018 & $<0.0001$ \\
\hline Inefficacy & 0 & & & \\
\hline \multicolumn{5}{|c|}{ For depression } \\
\hline Efficacy & -0.0269 & -0.039 & -0.0148 & $<0.0001$ \\
\hline Inefficacy & 0 & & & \\
\hline
\end{tabular}

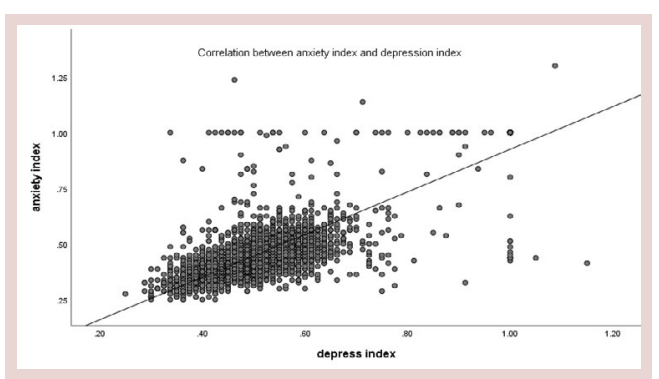

Figure 1. Anxiety index was associated with depression index.

inefficacy group had a higher anxiety index and depression index than patients in drug efficacy group.

Although the mechanisms by which drug efficacy impact psychological status are not entirely clear, disease activity may play an important role in the association between psychological status and drug efficacy in patients with ankylosing spondylitis [12]. In this study, AS patients in drug inefficacy group have higher degree disease activity than patients in drug efficacy group while disease activity is suspected to be one of the main risk factors of psychological disorders in patients with AS [12]. In addition, drug inefficacy will increase medical costs for patients with AS, while anxiety and depression are significantly associated with increased medical costs $[21,22]$.

Furthermore, we compared anxiety index and depression index between drug efficacy group and drug inefficacy group for different drug therapy status. Anxiety index and depression index were significantly lower in efficacy group than inefficacy group for NSAIDs on demand, NSAIDs continued and SSZ. Very interestingly, there was no significant difference between efficacy group and inefficacy group for Etanercept. Consistent with a previous study [22], biologic agents may have an antidepressant efficacy besides their anti-inflammatory effect that is independent of their clinical effect.
From the above discussion, different drug therapy status will lead to different results for patients with AS. Therefore, we want to know whether the differences of anxiety index and depression index between drug efficacy group and drug inefficacy group contributes to the differences of drug therapy status. It is displayed in Table 1 that there is a significant difference in drug therapy status between these two groups. Therefore, in addition to adjust for sex, age and age of onset, we estimated the association between psychological status and drug efficacy after adjusting for different drug therapy status (NSAIDs on demand, NSAIDs continued, SSZ and Etanercept). Consistent with above results, the association between psychological status and drug efficacy was still significant after adjusting for sex, age, age of onset and different drug therapy status.

This study has several strengths, especially the use of large sample size with high representativity. However, there are several limitations in this study. This is a cross-sectional observational study, which cannot build a causal association between psychological status and drug efficacy. This is a cross-sectional observational study; it cannot build a causal association between psychological status and drug efficacy. Although drug therapy inefficacy can lead to anxiety and depression, anxiety and depression may lead to drug therapy inefficacy. In addition, many risk factors, such as disease activity, functional disability, socioeconomic status and lack of health insurance are not taken into account in this study.

\section{Conclusions}

In summary, these findings from the study indicate that there is a significant association between psychological status and drug efficacy in patients with ankylosing spondylitis. Our results have potential implications on clinical assessment and management of ankylosing spondylitis. 


\section{References}

1. Van der Heijde D, Breban M, Halter D et al. Maintenance of improvement in spinal mobility, physical function and quality of life in patients with ankylosing spondylitis after 5 years in a clinical trial of adalimumab. Rheumatology (Oxford). 54(7), 12101219 (2015).

2. Dean LE, Jones GT, MacDonald AG et al. Global prevalence of ankylosing spondylitis. Rheumatology (Oxford). 53(4), 650-657 (2014).

3. Lehtinen K. Mortality and causes of death in 398 patients admitted to hospital with ankylosing spondylitis. Ann. Rheum. Dis. 52(3), 174-176 (1993).

4. Greenberg JD, Palmer JB, Li Y et al. Healthcare Resource Use and Direct Costs in Patients with Ankylosing Spondylitis and Psoriatic Arthritis in a Large US Cohort. J. Rheumatol. 43(1), 88-96 (2016).

5. Malinowski KP, Kawalec P. The indirect costs of ankylosing spondylitis: a systematic review and metaanalysis. Expert. Rev. Pharmacoecon. Outcomes. Res. 15(2), 285-300 (2015).

6. Lee TJ, Park BH, Kim JW et al. Cost-of-illness and quality of life in patients with ankylosing spondylitis at a tertiary hospital in Korea. J. Korean. Med. Sci. 29(2), 190-197 (2014)

7. Maksymowych WP, Richardson R, Mallon C et al. Evaluation and validation of the patient acceptable symptom state (PASS) in patients with ankylosing spondylitis. Arthritis. Rheum. 57(1), 133-139 (2007).

8. Spoorenberg A, van Tubergen A, Landewé $\mathrm{R}$ et al. Measuring disease activity in ankylosing spondylitis: patient and physician have different perspectives. Rheumatology (Oxford). 44(6), 789-795 (2005).

9. Hakkou J, Rostom S, Aissaoui $\mathrm{N}$ et al. Psychological status in Moroccan patients with ankylosing spondylitis and its relationships with disease parameters and quality of life. J. Clin. Rheumatol. 17(8), 424-428 (2011).

10. Baysal O, Durmuş B, Ersoy Y et al. Relationship between psychological status and disease activity and quality of life in ankylosing spondylitis. Rheumatol. Int. 31(6), 795-800 (2011).

11. Xu X, Shen B, Zhang A et al. Anxiety and depression correlate with disease and quality-of-life parameters in Chinese patients with ankylosing spondylitis. Patient. Prefer. Adherence. 10, 879-885 (2016).
12. Martindale J, Smith J, Sutton CJ et al. Disease and psychological status in ankylosing spondylitis. Rheumatology (Oxford). 45(10), 1288-1293 (2006).

13. Batmaz İ, Sarıyıldız MA, Dilek B et al. Sleep quality and associated factors in ankylosing spondylitis: relationship with disease parameters, psychological status and quality of life. Rheumatol. Int. 33(4), 10391045 (2013).

14. Hermann B, Jones J. Intractable epilepsy and patterns of psychiatric comorbidity. Adv. Neurol. 97, 367-374 (2006).

15. Devinsky O, Barr WB, Vickrey BG et al. Changes in depression and anxiety after resective surgery for epilepsy. Neurology. 65(11), 1744-1749 (2005).

16. Meldolesi GN, Di Gennaro G, Quarato PP et al. Changes in depression, anxiety, anger, and personality after respective surgery for drug-resistant temporal lobe epilepsy: a 2-year follow-up study. Epilepsy. Res. 77(1), 22-30 (2007).

17. Kiltz U, Heldmann F, Baraliakos X et al. Treatment of ankylosing spondylitis in patient's refractory to TNF-inhibition: are there alternatives? Curr. Opin. Rheumatol. 24(3), 252-260 (2012).

18. Van der Linden S, Valkenburg HA et al. Evaluation of diagnostic criteria for ankylosing spondylitis. A proposal for modification of the New York criteria. Arthritis. Rheum. 27(4), 361-368 (1984).

19. Lindsay WR, Michie AM. Adaptation of the Zung selfrating anxiety scale for people with a mental handicap. J. Ment. Defic. Res. 32(6), 485-490 (1988).

20. Epkins CC, Meyers AW. Assessment of childhood depression, anxiety, and aggression: convergent and discriminant validity of self, parent, teacher and peerreport measures. J. Pers. Assess. 62(2), 64-81 (1994).

21. Peńa P, Sancho J, Rufo M et al. LINCE Study Collaborative Group. Driving cost factors in adult outpatients with refractory epilepsy: a daily clinical practice in clinics of neurology in Spain. Epilepsy. Res. 83(2-3), 133-143 (2009).

22. Arısoy O, Bes C, Cifci $\mathrm{C}$ et al. The efficacy of TNFalpha blockers on psychometric measures in ankylosing spondylitis patients: a preliminary observation. Rheumatol. Int. 33(7), 1855-1864 (2013). 